\title{
Possible Gender Difference in the Association Between Abdominal Obesity, Chronic Inflammation, and Preclinical Atherosclerosis in the General Population
}

\author{
Tatsuya Kamon, ${ }^{1} \mathrm{MD}$, Hidehiro Kaneko, ${ }^{1,2} \mathrm{MD}$, Hidetaka Itoh, ${ }^{1} \mathrm{MD}$, Hiroyuki Kiriyama, ${ }^{1} \mathrm{MD}$, \\ Yoshiko Mizuno, ${ }^{1,3}$ MD, Hiroyuki Morita, ${ }^{1}$ MD, Norifumi Takeda, ${ }^{1}$ MD, \\ Nobutake Yamamichi, ${ }^{3}$ MD and Issei Komuro, ${ }^{1}$ MD
}

\begin{abstract}
Summary
Chronic inflammation due to abdominal obesity plays a major role in the development of cardiovascular disease (CVD). Gender differences are well characterized in the development of CVD; however, in the association among abdominal obesity, chronic inflammation, and preclinical atherosclerosis, gender differences in the general population remain to be clarified. We retrospectively analyzed 1,163 subjects who underwent voluntary health checkups at our institute. We defined carotid artery plaque formation as carotid intima-media thickness $\geq$ $1.1 \mathrm{~mm}$. Multiple regression analysis showed that waist circumference was a major independent predictor of increase in serum C-reactive protein (CRP) level in both men and women. Serum CRP level was significantly increased in men with carotid artery plaque formation, but not in women. Multivariable logistic regression analysis demonstrated that serum CRP level, as well as age and hypertension, was independently associated with carotid artery plaque formation only in men. This result may suggest a potential of gender-specific difference in the association between serum CRP level and the prevalence of carotid artery plaque formation. Further investigations are required to confirm our results and to clarify the underlying mechanism.
\end{abstract}

(Int Heart J 2021; 62: 837-842)

Key words: Waist circumference, C-reactive protein, Carotid intima-media thickness

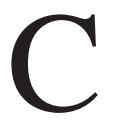

hronic inflammation orchestrates the pathological relationship between abdominal obesity due to visceral fat accumulation and the development of cardiovascular disease $(\mathrm{CVD}){ }^{1-6)}$ Modulating chronic inflammation is considered an important strategy for the prevention of CVD in the general population.

Health checkups, which are mainly performed for the early detection of lifestyle-related diseases and primary prevention of CVD, involve measurement of waist circumference (WC) for the assessment of abdominal obesity ${ }^{7,8}$ and $\mathrm{C}$-reactive protein (CRP) as a marker of chronic inflammation. Additionally, carotid artery ultrasound is performed to assess carotid artery plaque formation, and it is a common technique used for the evaluation of preclinical atherosclerosis in the general population. ${ }^{9-13)}$

Gender differences should be considered from the viewpoint of primary prevention. Men are at higher risk of CVD ${ }^{14-16)}$ and CVD risk factors promote CVD in both genders, albeit with different relative importance. Abdominal obesity and chronic inflammation are closely related to the development of CVD; however, gender differences in the association between abdominal obesity, chronic inflammation, and preclinical atherosclerosis in the general population remain to be clarified.

In this study, we examined subjects who underwent voluntary health checkups and sought to clarify whether there is a gender-specific difference in the relationship between WC, CRP, and the prevalence of carotid artery plaque formation.

\section{Methods}

Study population: We examined 1,243 subjects who underwent medical checkups at the University of Tokyo Hospital from August 2014 to May 2018. We analyzed the collected data between August and October 2018. We included subjects aged $\geq 18$ years who were willing to participate in the retrospective study and excluded 2 subjects whose carotid intima-media thickness (IMT) data were unavailable; 59 subjects with a past history of coronary artery disease $(\mathrm{CAD})$, stroke, and peripheral arterial disease; and 19 subjects whose CRP level is $\geq 1 \mathrm{mg} / \mathrm{dL}^{.17)}$ Finally, a total of 1,163 subjects were included in this study. Study subjects were divided into two groups, based on gender (flowchart is shown in Figure 1).

Ethics: This study was conducted in accordance with the

From the ${ }^{1}$ Department of Cardiovascular Medicine, School of Medicine, The University of Tokyo, Tokyo, Japan, ${ }^{2}$ Department of Advanced Cardiology, Graduate School of Medicine, The University of Tokyo, Tokyo, Japan and ${ }^{3}$ Center for Epidemiology and Preventive Medicine, The University of Tokyo Hospital, Tokyo, Japan.

Address for correspondence: Hidehiro Kaneko, MD, Department of Cardiovascular Medicine, The University of Tokyo Hospital, 7-3-1, Hongo, Bunkyo-ku, Tokyo, 113-8655, Japan. E-mail: kanekohidehiro@gmail.com

Received for publication September 26, 2020. Revised and accepted January 6, 2021.

doi: $10.1536 /$ ihj.20-654

All rights reserved by the International Heart Journal Association. 


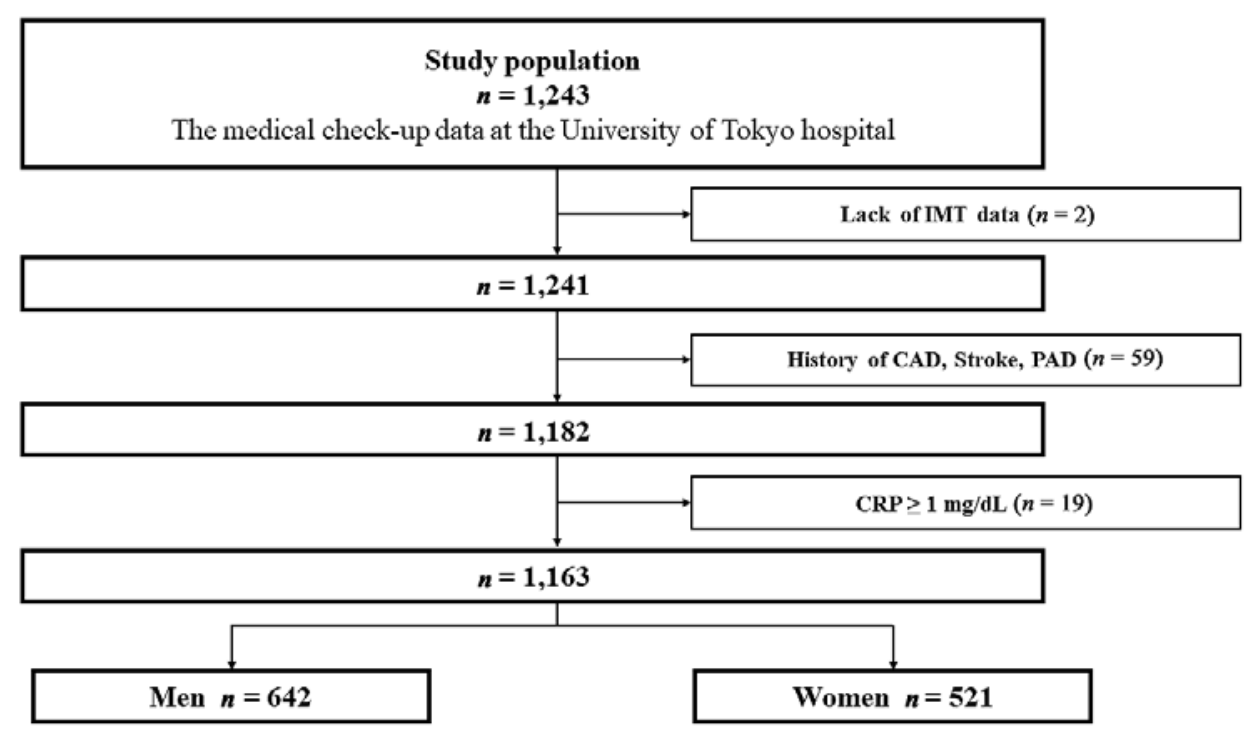

Figure 1. Flowchart of study population. We excluded 2 subjects whose IMT data were unavailable; 59 subjects with a past history of CAD, stroke, and PAD; and 19 subjects with CRP $\geq 1 \mathrm{mg} / \mathrm{dL}$. Finally, 1,163 subjects were included in this study. IMT, intima-media thickness; CAD, coronary artery disease; PAD, peripheral artery disease; CRP, C-reactive protein.

ethical guidelines of our institution (approval by the Ethical Committee of The University of Tokyo: 2017-2424) and the principles of the Declaration of Helsinki. Informed consent was obtained from all individuals enrolled in this study using the opt-out procedure.

Measurement of WC and carotid plaque formation: WC was measured at the level of the umbilicus with a non-stretchable tape in late expiration while standing. Carotid IMT was measured using a B-mode ultrasound imager (Aplio 300, Toshiba, Tokyo, Japan). The bilateral carotid artery was examined with a 7.5 $\mathrm{MHz}$ probe. The diameter of the internal lumen was calculated by manual tracing along the same length as the carotid IMT and averaging the distances between the near and far lumenintima interfaces. The average of the right and left side measurements was used to calculate the carotid IMT. We defined carotid artery plaque formation as carotid IMT $\geq$ $1.1 \mathrm{~mm}$, as previously reported. ${ }^{18,19)}$

Clinical and laboratory analysis: We collected information regarding the presence of cardiovascular risk factors, including hypertension, diabetes mellitus (DM), and hypercholesterolemia, from medical records of each subject and self-administrated questionnaires. Blood was collected during fasting to determine the glucose, hemoglobin A1c, total cholesterol, low-density lipoprotein cholesterol, highdensity lipoprotein cholesterol, triglycerides, and CRP levels. We defined obesity as body mass index (BMI) $\geq 25$ $\mathrm{kg} / \mathrm{m}^{2}$. Hypertension was defined as a blood pressure $\geq 140 / 90 \mathrm{mmHg}$ or use of antihypertensive medications. $\mathrm{DM}$ was defined as fasting glucose level $\geq 126 \mathrm{mg} / \mathrm{dL}$ or use of oral antidiabetic medications or insulin. Hypercholesterolemia was defined as a total serum cholesterol > $240 \mathrm{mg} / \mathrm{dL}$ or use of antihyperlipidemic medications.

Statistical analysis: Categorical and consecutive data of the baseline characteristics are presented as percentages (\%) and means \pm standard deviation. The chi-square test was used to compare the categorical variables. The unpaired $t$-test was used to compare consecutive variables. Multiple regression analysis was used to analyze the relationship between CRP and clinical variables. We performed multivariable logistic regression analysis for the parameters that could affect carotid IMT, including age, obesity, WC, hypertension, DM, hypercholesterolemia, smoking, and CRP. The $P$-values for interactions between gender groups in the association of CRP with carotid IMT were calculated. A probability value of $<0.05$ was considered statistically significant. We performed all statistical analyses using SPSS (SPSS Inc., Chicago, IL, USA) version 25 software.

\section{Results}

Baseline characteristics: Table I summarizes the baseline characteristics of the study subjects. In total, 1,163 subjects (642 men and 521 women, $62.2 \pm 11.7$ years on average) were analyzed in this study. CRP on average was $0.09 \pm 0.13 \mathrm{mg} / \mathrm{dL}$. Overall, carotid IMT was $1.0 \pm 0.3$ $\mathrm{mm}$ on average, and carotid artery plaque formation was observed in $36.5 \%$ of study subjects.

Correlation of CRP with clinical variables analyzed by multiple regression model: Table II summarizes the results of a multiple regression analysis of the correlation of CRP with clinical variables. WC was an independent determinant of CRP in both men and women.

CRP and carotid plaque formation: Serum CRP level was significantly higher in men with carotid artery plaque formation than in those without $(0.11 \pm 0.15 \mathrm{mg} / \mathrm{dL}$ versus $0.09 \pm 0.11 \mathrm{mg} / \mathrm{dL}, P=0.022$, Figure $2 \mathrm{~A}$ ), but not in women $(0.08 \pm 0.12 \mathrm{mg} / \mathrm{dL}$ versus $0.08 \pm 0.11 \mathrm{mg} / \mathrm{dL}, P$ $=0.469$, Figure 2B).

Determinants of carotid plaque formation: Multivariable logistic regression analysis demonstrated that CRP 
Table I. Baseline Clinical Characteristics

\begin{tabular}{|c|c|c|c|c|}
\hline Variable & $\begin{array}{c}\text { Overall } \\
(n=1,163)\end{array}$ & $\begin{array}{c}\text { Men } \\
(n=642)\end{array}$ & $\begin{array}{l}\text { Women } \\
(n=521)\end{array}$ & $P$-value \\
\hline Age (year) & $62.2 \pm 11.7$ & $61.2 \pm 11.7$ & $63.4 \pm 11.5$ & 0.001 \\
\hline Body mass index $\left(\mathrm{kg} / \mathrm{m}^{2}\right)$ & $23.5 \pm 3.6$ & $24.5 \pm 3.4$ & $22.3 \pm 3.5$ & $<0.001$ \\
\hline Obesity & $353(30.4 \%)$ & $255(39.7 \%)$ & $98(18.8 \%)$ & $<0.001$ \\
\hline Waist circumference & $83.8 \pm 10.0$ & $86.8 \pm 9.1$ & $80.0 \pm 9.7$ & $<0.001$ \\
\hline Hypertension & $405(34.8 \%)$ & $249(38.8 \%)$ & $156(29.9 \%)$ & 0.002 \\
\hline Diabetes mellitus & $116(10.0 \%)$ & $93(14.5 \%)$ & $23(4.4 \%)$ & $<0.001$ \\
\hline Hypercholesterolemia & $423(36.4 \%)$ & $204(31.8 \%)$ & $219(42.0 \%)$ & $<0.001$ \\
\hline Smoking & $434(37.3 \%)$ & $342(53.3 \%)$ & $92(17.7 \%)$ & $<0.001$ \\
\hline Glucose (mg/dL) & $99.8 \pm 20.2$ & $103.9 \pm 22.8$ & $94.8 \pm 15.1$ & $<0.001$ \\
\hline $\operatorname{HbA} 1 \mathrm{c}(\%)$ & $5.8 \pm 0.6$ & $5.9 \pm 0.7$ & $5.8 \pm 0.5$ & $<0.001$ \\
\hline T-cholesterol (mg/dL) & $206.2 \pm 34.1$ & $200.5 \pm 33.3$ & $213.2 \pm 33.8$ & $<0.001$ \\
\hline LDL-C (mg/dL) & $125.0 \pm 30.4$ & $123.8 \pm 30.7$ & $126.4 \pm 29.9$ & 0.153 \\
\hline HDL-C (mg/dL) & $65.7 \pm 18.7$ & $59.0 \pm 15.5$ & $73.9 \pm 18.9$ & $<0.001$ \\
\hline $\mathrm{TG}(\mathrm{mg} / \mathrm{dL})$ & $111.0 \pm 79.8$ & $127.0 \pm 92.3$ & $91.2 \pm 54.9$ & $<0.001$ \\
\hline $\mathrm{CRP}(\mathrm{mg} / \mathrm{dL})$ & $0.09 \pm 0.13$ & $0.10 \pm 0.13$ & $0.08 \pm 0.12$ & 0.021 \\
\hline Carotid IMT (mm) & $1.0 \pm 0.3$ & $1.1 \pm 0.3$ & $1.0 \pm 0.3$ & $<0.001$ \\
\hline Carotid IMT $\geq 1.1 \mathrm{~mm}$ & $424(36.5 \%)$ & $274(42.7 \%)$ & $150(28.8 \%)$ & $<0.001$ \\
\hline
\end{tabular}

Data are expressed as mean \pm standard deviation or number (percentage). HbAlc indicates hemoglobin Alc; LDL-C, low-density lipoprotein cholesterol; HDL-C, high-density lipoprotein cholesterol; TG, triglycerides; CRP, C-reactive protein; and IMT, intima-media thickness.

Table II. Correlation of CRP with Clinical Variables

\begin{tabular}{lccrrrr}
\hline Variable & \multicolumn{3}{c}{ Men } & \multicolumn{3}{c}{ Women } \\
& $\beta$ coefficient & SE & $P$-value & $\beta$ coefficient & \multicolumn{1}{c}{ SE } & $P$-value \\
\hline Age & 0.002 & $<0.001$ & 0.969 & 0.006 & $<0.001$ & 0.904 \\
Obesity & -0.059 & 0.015 & 0.274 & 0.080 & 0.017 & 0.152 \\
Waist circumference & 0.180 & 0.001 & 0.001 & 0.292 & 0.001 & $<0.001$ \\
Hypertension & 0.084 & 0.011 & 0.049 & 0.017 & 0.011 & 0.704 \\
Diabetes mellitus & -0.049 & 0.015 & 0.232 & -0.042 & 0.025 & 0.328 \\
Hypercholesterolemia & -0.031 & 0.011 & 0.441 & -0.089 & 0.010 & 0.039 \\
Smoking & -0.008 & 0.010 & 0.840 & 0.002 & 0.013 & 0.969 \\
\hline
\end{tabular}

$\mathrm{CRP}$ indicates $\mathrm{C}$-reactive protein; and SE, standard error.

level, as well as age and hypertension, was independently associated with carotid artery plaque formation in men. However, CRP level was not independently associated with carotid artery plaque formation in women (Table III). The association of CRP with carotid artery plaque formation was not modified by gender (the $P$ for interaction between gender was 0.385 ). Obesity and WC were not associated with carotid artery plaque formation in men and women, whereas DM was independently associated with carotid artery plaque formation only in women.

\section{Discussion}

There are three major findings in this study. First, high WC is associated with elevated CRP levels in both men and women. Second, serum CRP level is higher in subjects with carotid artery plaque formation than in those without, in men but not in women. Third, elevated CRP level is independently associated with the prevalence of carotid artery plaque formation only in men.

Abdominal obesity, which is caused by visceral fat accumulation, causes chronic systemic inflammation and is known to lead to subsequent CVD. Elevated serum CRP level is widely used not only as a marker of chronic inflammation but also as a predictor of future CVD events. ${ }^{20)}$ Preceding studies showed that a high level of serum CRP is a marker of carotid atherosclerosis among subjects with CVD risk. ${ }^{21)}$ Curb, et al. reported that a high level of serum CRP was positively associated with thromboembolic stroke in men aged 48-70 years and with no history of CVD. ${ }^{22)}$ Ridker, et al. also reported that an elevated CRP level was associated with the increased incidence of myocardial infarction and stroke in subjects with no history of CVD, and the use of aspirin, which has both antithrombotic and anti-inflammatory effects, reduced the risk of myocardial infarction. ${ }^{23)}$

There is a substantial gender difference in the development of atherosclerotic CVD. Previous studies identified male gender as an independent risk factor for higher rates of CVD and CVD-related mortality. ${ }^{14-16)}$ Anand, et al. showed that women experience their first acute myocardial infarction on average 9 years later than men in INTERHEART global case-control study. ${ }^{24)}$ CVD risk factors promote CVD in both genders, but with different relative importance. For example, clear gender difference was observed in the impact of DM and smoking for the development of CVD. ${ }^{25-28)}$ Juutilainen, et al. reported considerably higher diabetes-related relative risk for a major CVD 
A

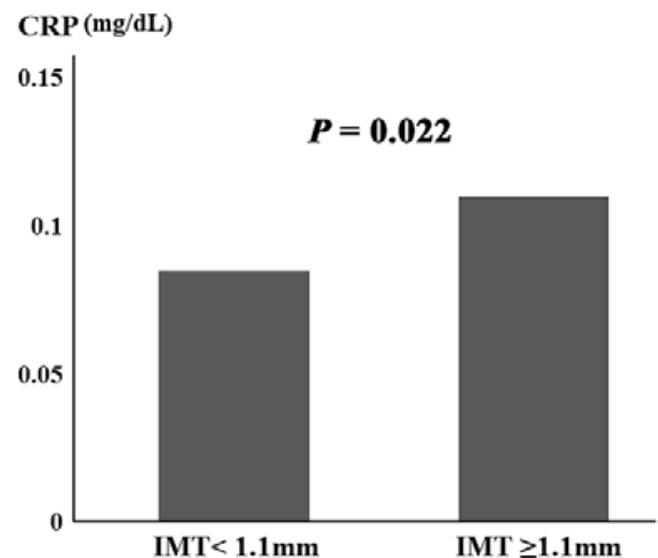

B

CRP (mg/dL)

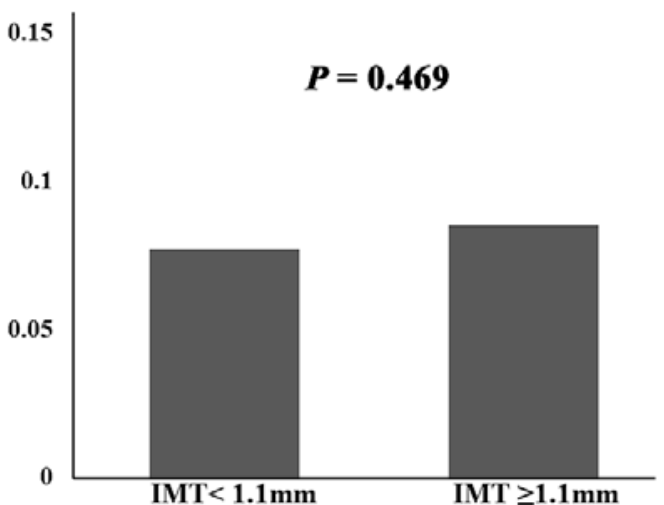

Figure 2. C-reactive protein and carotid plaque formation. Serum CRP level was significantly higher in subjects with carotid artery plaque formation than those without in men (A), but not in women (B). IMT, intima-media thickness; CRP, C-reactive protein.

Table III. Determinants of Carotid Artery Plaque Formation

\begin{tabular}{lrllrrr}
\hline \multicolumn{1}{c}{ Variable } & \multicolumn{3}{c}{ Men } & \multicolumn{3}{c}{ Women } \\
& $P$-value & OR & $95 \%$ CI & $P$-value & OR & $95 \%$ CI \\
\hline Age & $<0.001$ & 1.1 & $1.1-1.1$ & $<0.001$ & 1.1 & $1.0-1.1$ \\
Obesity & 0.235 & 1.3 & $0.8-2.2$ & 0.828 & 1.1 & $0.5-2.2$ \\
Waist circumference & 0.198 & 1.0 & $1.0-1.0$ & 0.627 & 1.0 & $1.0-1.0$ \\
Hypertension & 0.043 & 1.5 & $1.0-2.2$ & 0.004 & 1.9 & $1.2-3.0$ \\
Diabetes mellitus & 0.285 & 1.3 & $0.8-2.2$ & 0.020 & 3.3 & $1.2-9.0$ \\
Hypercholesterolemia & 0.465 & 1.2 & $0.8-1.7$ & 0.391 & 1.2 & $0.8-1.8$ \\
Smoking & 0.988 & 1.0 & $0.7-1.4$ & 0.106 & 0.6 & $0.3-1.1$ \\
C-reactive protein & 0.038 & 4.3 & $1.1-16.9$ & 0.982 & 1.0 & $0.2-6.4$ \\
\hline
\end{tabular}

OR indicates odds ratio; and CI, confidence interval.

event in diabetic women than in diabetic men among subjects aged 45-64 years without a history of CVD. ${ }^{29)}$ Loboz-Rudnicka, et al. showed that the impact of DM on IMT thickening is greater in women than in men. ${ }^{30)}$ In this study, DM was associated with carotid artery plaque formation only in women as well.

However, the possible gender difference in the association between abdominal obesity, chronic inflammation, and preclinical atherosclerosis is not well investigated. In this study, we examined 1,163 subjects (642 men and 521 women) who underwent voluntary health checkups at our institute. We evaluated abdominal obesity by WC and assessed chronic inflammation using serum CRP level. Among both men and women, high WC was independently associated with elevated CRP level, suggesting that there is no obvious gender difference in the association between abdominal obesity and chronic inflammation. However, multivariable logistic regression analysis showed that CRP level was an independent determinant of carotid artery plaque formation as well as age and hypertension in men, whereas CRP level was not independently associated with carotid artery plaque formation in women. However, given the $P$ for interaction, we could not conclude that the influence of $\mathrm{CRP}$ on carotid artery plaque formation would be different between men and women. Further studies with larger sample size are required to confirm our results and to conclude the possible gender difference in the relationship between CRP and carotid artery plaque formation.

Considering that high serum CRP level was associated with carotid artery plaque formation in men, abdominal obesity may lead to preclinical atherosclerosis mainly driven by chronic inflammation among men. Although elevated CRP was not independently associated with carotid artery plaque formation in women, previous studies indicated that elevated CRP is useful in predicting CVD not only in men but also in women. ${ }^{31-34)}$ There are several possible explanations as to why serum CRP level was not associated with carotid artery plaque formation in women in our study. There are known substantial racial differences in baseline chronic inflammation. For example, it has been reported that the serum CRP baseline level is $0.20 \mathrm{mg} / \mathrm{dL}$ in White and $0.26 \mathrm{mg} / \mathrm{dL}$ in Black residents of the United States, whereas it is $0.10 \mathrm{mg} / \mathrm{dL}$ in East Asians. ${ }^{35)}$ Accordingly, the average serum CRP level in our study was $0.10 \mathrm{mg} / \mathrm{dL}$ in men and $0.08 \mathrm{mg} / \mathrm{dL}$ in women. Low baseline level of inflammation may affect the results. Furthermore, we investigated subjects who underwent voluntary health checkups; hence, our cohort may include more subjects at low risk of CVD than preceding 
studies focusing on the role of chronic inflammation in the development of subsequent CVD. This background might also influence the result. Additionally, our cohort included premenopausal women (the exact number is unknown), and female hormones, including estrogen, are known to have various protective effects against atherosclerosis, which could also affect the result of the present study. We conducted a subgroup analysis including women aged $\geq 60$ years to investigate the effects of menopause. Even in this analysis, we failed to find the association between CRP and carotid artery plaque formation. However, due to the limited sample size, we could not draw a solid conclusion regarding this point. Thus, further data are needed on this perspective.

We believe that our results have clinical implications. Currently, abdominal obesity, chronic inflammation, and the development of CVD are considered as a central axis of lifestyle-related disease and subsequent CVD. Accordingly, various studies reported the potential of interventions targeting chronic inflammation. The JUPITER trial showed that rosuvastatin significantly reduced the incidence of major cardiovascular events in healthy men and women with elevated serum CRP levels. ${ }^{36)}$ Furthermore, the CANTOS trial indicated that monoclonal antibody targeting interleukin-1 $\beta$ reduced the future cardiovascular events in subjects with previous myocardial infarction and a CRP level of $0.2 \mathrm{mg} / \mathrm{dL}$ or more. ${ }^{37)}$ Given these studies, modulating chronic inflammation has been recognized as a key strategy for the prevention of CVD. However, considering the results of the present study, we may need to reconsider the validity of this concept, particularly in women.

This study has several limitations. First, this study was based on a single-center cohort. Therefore, it is not easy to generalize our results. Due to the limited sample size, the statistical power is not sufficient for any nonsignificant data to be conclusive. Second, a cause-effect relationship could not be examined due to the cross-sectional design of this study. Although we performed multivariable analysis, the possibility of unmeasured confounders could not be excluded. Third, we did not know the menopausal status of women. Thus, we could not investigate the impact of menopause on the development of atherosclerosis. Fourth, this is a cross-sectional study, and serial change in LMT was not evaluated in this study. Therefore, it is not possible to conclude gender differences in the effect of chronic inflammation on the development of atherosclerosis based on this study alone.

\section{Conclusions}

In conclusion, high WC was associated with elevated CRP levels in men and women. High WC was associated with the prevalence of carotid artery plaque formation neither in men nor in women. Elevated CRP level is independently associated with the prevalence of carotid artery plaque formation in men but not in women. Further investigations are required to confirm our results and to assess the possible gender difference in the association between chronic inflammation and preclinical atherosclerosis.

\section{Acknowledgments}

We thank the staff of the Center for Epidemiology and Preventive Medicine in our institute.

\section{Disclosure}

Conflicts of interest: The authors declare no conflict of interest.

\section{References}

1. Hansson GK. Inflammation, atherosclerosis, and coronary artery disease. N Engl J Med 2005; 352: 1685-95.

2. Libby P, Ridker PM, Hansson GK, Leducq Transatlantic Network on Atherothrombosis. Inflammation in atherosclerosis: from pathophysiology to practice. J Am Coll Cardiol 2009; 54: 2129-38.

3. Libby P. Inflammation in atherosclerosis. Nature 2002; 420: 868-74.

4. Willerson JT, Ridker PM. Inflammation as a cardiovascular risk factor. Circulation 2004; 109: II2-10.

5. Fontana L, Eagon JC, Trujillo ME, Scherer PE, Klein S. Visceral fat adipokine secretion is associated with systemic inflammation in obese humans. Diabetes 2007; 56: 1010-3.

6. Mathieu P, Lemieux I, Després JP. Obesity, inflammation, and cardiovascular risk. Clin Pharmacol Ther 2010; 87: 407-16.

7. The Examination Committee of Criteria for 'Obesity Disease' in Japan, Japan Society for the Study of Obesity. New criteria for 'obesity disease' in Japan. Circ J 2002; 66: 987-92.

8. Hiuge-Shimizu A, Kishida K, Funahashi T, et al. Absolute value of visceral fat area measured on computed tomography scans and obesity-related cardiovascular risk factors in large-scale Japanese general population (the VACATION-J study). Ann Med 2012; 44: 82-92.

9. de Groot E, Hovingh GK, Wiegman A, et al. Measurement of arterial wall thickness as a surrogate marker for atherosclerosis. Circulation 2004; 109: III33-8.

10. Kitamura A, Iso $\mathrm{H}$, Imano $\mathrm{H}$, et al. Carotid intima-media thickness and plaque characteristics as a risk factor for stroke in Japanese elderly men. Stroke 2004; 35: 2788-94.

11. Rosvall M, Janzon L, Berglund G, Engström G, Hedblad B. Incidence of stroke is related to carotid IMT even in the absence of plaque. Atherosclerosis 2005; 179: 325-31.

12. Rosvall M, Janzon L, Berglund G, Engstrom G, Hedblad B. Incident coronary events and case fatality in relation to common carotid intima-media thickness. J Intern Med 2005; 257: 430-7.

13. Bots ML, Baldassarre D, Simon A, et al. Carotid intima-media thickness and coronary atherosclerosis: weak or strong relations? Eur Heart J 2007; 28: 398-406.

14. Tunstall-Pedoe H, Kuulasmaa K, Mähönen M, Tolonen H, Ruokokoski E, Amouyel P. Contribution of trends in survival and coronary-event rates to changes in coronary heart disease mortality: 10-year results from 37 WHO MONICA project populations. Monitoring trends and determinants in cardiovascular disease. Lancet 1999; 353: 1547-57.

15. D'Agostino RB Sr, Vasan RS, Pencina MJ, et al. General cardiovascular risk profile for use in primary care: the Framingham heart study. Circulation 2008; 117: 743-53.

16. Kappert K, Böhm M, Schmieder R, et al. Impact of sex on cardiovascular outcome in patients at high cardiovascular risk: analysis of the telmisartan randomized assessment study in ACE-intolerant subjects with cardiovascular disease (TRANSCEND) and the ongoing telmisartan alone and in combination with ramipril global end point trial (ONTARGET). Circulation 2012; 126: 934-41.

17. Tomiyama H, Okazaki R, Koji Y, et al. Elevated C-reactive pro- 
tein: a common marker for atherosclerotic cardiovascular risk and subclinical stages of pulmonary dysfunction and osteopenia in a healthy population. Atherosclerosis 2005; 178: 187-92.

18. Salonen R, Salonen JT. Progression of carotid atherosclerosis and its determinants: a population-based ultrasonography study. Atherosclerosis 1990; 81: 33-40.

19. Itoh H, Kaneko H, Kiriyama $\mathrm{H}$, et al. Relation between the updated blood pressure classification according to the American College of Cardiology/American Heart Association guidelines and carotid intima-media thickness. Am J Cardiol 2019; 124 396-401.

20. Danesh J, Wheeler JG, Hirschfield GM, et al. C-reactive protein and other circulating markers of inflammation in the prediction of coronary heart disease. N Engl J Med 2004; 350: 1387-97.

21. Hashimoto H, Kitagawa K, Hougaku H, et al. C-reactive protein is an independent predictor of the rate of increase in early carotid atherosclerosis. Circulation 2001; 104: 63-7.

22. Curb JD, Abbott RD, Rodriguez BL, et al. C-reactive protein and the future risk of thromboembolic stroke in healthy men Circulation 2003; 107: 2016-20.

23. Ridker PM, Cushman M, Stampfer MJ, Tracy RP, Hennekens $\mathrm{CH}$. Inflammation, aspirin, and the risk of cardiovascular disease in apparently healthy men. N Engl J Med 1997; 336: $973-$ 9.

24. Anand SS, Islam S, Rosengren A, et al. Risk factors for myocardial infarction in women and men: insights from the INTERHEART study. Eur Heart J 2008; 29: 932-40.

25. Fox CS, Coady S, Sorlie PD, et al. Trends in cardiovascular complications of diabetes. JAMA 2004; 292: 2495-9.

26. Fox CS, Pencina MJ, Wilson PWF, Paynter NP, Vasan RS, D'Agostino RB Sr. Lifetime risk of cardiovascular disease among individuals with and without diabetes stratified by obesity status in the Framingham heart study. Diabetes Care 2008 31: 1582-4.

27. Prescott E, Hippe M, Schnohr P, Hein HO, Vestbo J. Smoking and risk of myocardial infarction in women and men: longitudinal population study. BMJ 1998; 316: 1043-7.
28. Njølstad I, Arnesen E, Lund-Larsen PG. Smoking, serum lipids, blood pressure, and sex differences in myocardial infarction: a 12-year follow-up of the Finnmark study. Circulation 1996; 93: 450-6.

29. Juutilainen A, Kortelainen S, Lehto S, Rönnemaa T, Pyörälä K, Laakso M. Gender difference in the impact of type 2 diabetes on coronary heart disease risk. Diabetes Care 2004; 27: 2898904.

30. Loboz-Rudnicka M, Jaroch J, Bociaga Z, et al. Impact of cardiovascular risk factors on carotid intima-media thickness: sex differences. Clin Interv Aging 2016; 11: 721-31.

31. Everett BM, Kurth T, Buring JE, Ridker PM. The relative strength of C-reactive protein and lipid levels as determinants of ischemic stroke as compared with coronary heart disease in women. J Am Coll Cardiol 2006; 48: 2235-42.

32. Ridker PM, Buring JE, Cook NR, Rifai N. C-reactive protein, the metabolic syndrome, and risk of incident cardiovascular events: an 8-year follow-up of 14719 initially healthy American women. Circulation 2003; 107: 391-7.

33. Ridker PM, Hennekens CH, Buring JE, Rifai N. C-reactive protein and other markers of inflammation in the prediction of cardiovascular disease in women. N Engl J Med 2000; 342: 83643.

34. Ridker PM, Buring JE, Shih J, Matias M, Hennekens CH. Prospective study of C-reactive protein and the risk of future cardiovascular events among apparently healthy women. Circulation 1998; 98: 731-3.

35. Shah T, Newcombe P, Smeeth L, et al. Ancestry as a determinant of mean population C-reactive protein values: implications for cardiovascular risk prediction. Circ Cardiovasc Genet 2010; 3: 436-44

36. Ridker PM, Danielson E, Fonseca FAH, et al. Rosuvastatin to prevent vascular events in men and women with elevated Creactive protein. N Engl J Med 2008; 359: 2195-207.

37. Ridker PM, Everett BM, Thuren T, et al. Antiinflammatory therapy with canakinumab for atherosclerotic disease. $\mathrm{N}$ Engl J Med 2017; 377: 1119-31. 